\title{
Currículo, historia y justicia social. Estudio comparativo en América Latina
}

Curriculum, History and Social Justice. A Comparative Study in Latin America

Currículo, história e justiça social. Estudo comparativo na América Latina

\section{Sebastián Plá* (iD) orcid.org/0000-0001-8613-1607}

\section{Artículo de investigación}

Revista Colombiana de Educación, N.⒎ 71. Segundo semestre de 2016, Bogotá, Colombia.

Para citar: Plá, S. (2016). Currículo, historia y justicia social. Estudio comparativo en América Latina. Revista Colombiana de Educación, (71), 53-77.

Recibido: 22/02/16

Evaluado: 26/02/16

* Profesor de la Universidad Pedagógica Nacional en México y profesor de Enseñanza de la Historia en la Facultad de Filosofía y Letras de la Universidad Nacional Autónoma de México. Doctor en Pedagogía por la Universidad Nacional Autónoma de México y licenciado en Historia por la Universidad Iberoamericana. Correo electrónico: sebastianpla@gmail.com 


\section{Resumen}

El presente artículo compara las narraciones acerca de los procesos de conquista y colonización de los siglos xv y xvı en Chile, México, Ecuador y Bolivia en sus programas de estudio vigentes para la educación obligatoria. Lo hace desde los conceptos de estrategia afirmativa y estrategia transformativa de la filosofía política de Nancy Fraser. El objetivo es observar el potencial deconstructivo de las relaciones simbólicas de poder en las narraciones históricas y analizar qué proyecto educativo responde mejor a las exigencias de reconocimiento de los grupos subalternos como parte de la lucha por la justicia social en estos cuatro países latinoamericanos. Los resultados muestran que Chile y México tienen un proyecto curricular afirmativo basado en principios de justicia surgidos de ciertas interpretaciones del liberalismo social. Por el contrario, Ecuador en menor grado y Bolivia en mayor, son propuestas curriculares potencialmente transformativas basadas en luchas descoloniales por la justicia social. Es un estudio cualitativo, comparativo y de carácter documental.

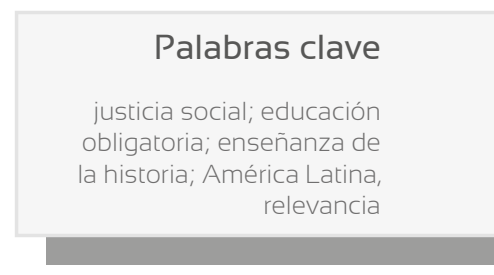

\section{Keywords}

social justice; compulsory education; history teaching; Latin America; relevance

\begin{abstract}
This article makes a comparative study of the historical narratives about the processes of conquest and colonization in the sixteenth and seventeenth centuries in current curricular programs for compulsory education in Chile, Mexico, Bolivia and Ecuador. The analysis is grounded in Nancy Fraser's concepts of affirmative and transformative strategies in political philosophy. The aim is to observe the deconstructive potential of symbolic power relations in historical narratives and analyze how each educational project responds to subaltern groups' demands for recognition, as part of the struggle for social justice in these four Latin American countries. The results show that Chile and Mexico have an affirmative currículum project based on principles of justice arising from social liberalism. Conversely Ecuador and, especially, Bolivia have potentially transformative curricula based on decolonial struggles for social justice. This is a qualitative, comparative and documentary study.
\end{abstract}

\section{Resumo}

O presente artigo compara as narrações sobre os processos da conquista e colonização dos séculos xv e xvı no Chile, México, Equador e Bolívia em seus programas de estudo vigentes para a educação obrigatória. O trabalha desde os conceitos de estratégia afirmativa e estratégia transformativa da filosofia política da Nancy Fraser. O objetivo é observar o potenciai deconstructivo das relações simbólicas do poder nas narrações históricas e analisar o que projeto educativo responde melhor ás exigências do reconhecimento dos grupos subalternos como parte da luta pela justiça social nestes quatro países latino-americanos. Os resultados mostram que o Chile e México tem um projeto curricular afirmativo com base nos princípios de justiça surgidos de certas interpretações do liberalismo social. Pelo contrário, Equador em menor grau e Bolívia em maior, são propostas curriculares potencialmente transformativas com base nas lutas decoloniais pela justiça social. É um estudo qualitativo, comparativo e documental.

\section{Palavras chave}

justiça social; educação obrigatória; ensinança da historia; América Latina; relevância 


\section{Introducción ${ }^{1}$}

I a educación como elemento de la justicia social y como agente para su consecución es algo que defienden tirios y troyanos. O en términos más contemporáneos y acordes a nuestra región, por descoloniales ${ }^{2}$ y neoliberales. Las causas se deben en parte a la maleabilidad que ofrece el tema para la demagogia, pero también a los principios políticos, económicos y filosóficos que componen las definiciones de justicia social y de educación. La pluralidad de enfoques se observa en la variedad temática y metodológica desde la que se ha estudiado el tema, aunque por lo general predominan los estudios cuantitativos que focalizan su energía en los tópicos de cobertura, exclusión, retención o eficiencia terminal entre otros. En menor grado se estudian las complejidades que implican los contenidos curriculares como problema de justicia social o, en su caso, la mirada hegemónica de los contenidos se concentra en la relación entre calidad educativa y resultados de aprendizaje. Estos estudios, en los que dominan los institutos nacionales de evaluación o los organismos internacionales dedicados a la evaluación, parten del supuesto de que el tipo de conocimiento que se evalúa es resultado de un consenso a nivel global. Ante el predominio de estas dos visiones, en especial de la última, considero importante problematizar el tipo de conocimiento que se prescribe y observar los procesos de exclusión simbólica que producen para llevar la relación entre justicia social y educación a derroteros comúnmente soslayados.

Problematizar los tipos de conocimiento que se prescriben en los sistemas educativos nacionales como parte de la justicia social tiene en los diseños curriculares una fuente primordial. Los planes y programas de estudio son un documento que incluye y excluye determinados contenidos y procesos cognitivos a partir de relaciones de poder entre los diversos actores (Goodson, 1995; Pinar, 2004) que lo definen. El resultado nunca es homogéneo y tiende a imbricar las fuerzas antagónicas bajo un consenso coyuntural para producir en las generaciones más jóvenes un sentido común acerca de las relaciones legítimas -y jerárquicas- entre saberes y grupos sociales dentro de una sociedad. En otras palabras, el currículo legitima las estructuras sociales, económicas, políticas y culturales hegemónicas y, por supuesto, las desigualdades materiales y simbólicas que las constituyen. Todas las asignaturas cumplen esta función, pero donde más

1 Este trabajo no hubiera sido posible sin el apoyo de Lucero San Vicente, Ixchel Romero, Ingrid de la Luz Bastida y Adolfo Ávila.

2 A lo largo del texto aparecerán los términos descolonial y decolonial. El primero hace referencia a las propuestas políticas y educativas de Ecuador y Bolivia, ya que lo utilizan así. El segundo hace referencia al movimiento teórico latinoamericano de autores como Aníbal Quijano, Arturo Escobar y Walter Mignolo entre otros. 
tangiblemente se puede ver es en las dedicadas a la enseñanza de las ciencias sociales, la historia, la geografía y la formación ciudadana. Por esto y para focalizar mi estudio, en el presente artículo me concentro en una sola: la historia.

Con base en lo anterior, las preguntas que guían mi investigación pueden formularse así: ¿Cómo reproducen o dislocan los contenidos históricos para la educación obligatoria las desigualdades simbólicas hegemónicas en una sociedad? Y ¿qué propuestas curriculares para la enseñanza de la historia se aproximan más, por lo menos potencialmente, a la educación como justicia social?

Para dar respuesta a estas preguntas he realizado un estudio de educación comparada sobre las narraciones históricas curriculares dedicadas a la conquista de América y a los procesos de colonización en cuatro países latinoamericanos: México, Chile, Bolivia y Ecuador. Los criterios de selección de estas repúblicas fueron dos: por un lado la continuidad y ruptura de los programas de estudio para la educación obligatoria actuales con el proceso de reformas educativas vivido en la última década del siglo xx en la región. México y Chile muestran mayor continuidad, mientras que Ecuador y Bolivia tratan de generar una ruptura. En segundo lugar se tomaron en cuenta los principios generales de justicia social que subyacen en los sistemas educativos. Chile y México son defensores de lo que podría denominarse liberalismo social e incluso neoliberal, mientras que los otros dos se pueden ubicar en visiones descoloniales de la justicia social. Esto permite establecer dos grupos fácilmente comparables. Por su parte, el contenido de conquista de América se seleccionó con base en tres criterios: (1) es un acontecimiento que sufrieron los cuatro países estudiados; (2) es un contenido histórico trabajado en los cuatro programas de estudio, y (3) es un contenido socialmente vivo (Legardez, 2003; Santisteban, 2010). Las fuentes utilizadas fueron varias, desde la Constitución Política de cada uno de estos cuatro países hasta los planes de estudio de primaria y educación media, libros de texto y guías para el docente.

La relación entre los contenidos históricos y la justicia social se estableció a través de las dimensiones de relevancia y pertinencia. La primera permite analizar el tipo de sociedad y de justicia social que subyace en las narraciones históricas curriculares y la segunda describe a qué tipo de sujeto social se le enseña y qué tipo se pretende formar. Tomando en cuenta ambas dimensiones, para comparar los contenidos se establecieron tres criterios sustentados en el reconocimiento de la diferencia como componente de la justicia o la injusticia social: la subalteridad de los pueblos indios, la conquista de América dentro de la historia universal y el binomio identidad/diversidad en las identidades nacionales. Finalmente, se valora el potencial para la justicia social de los contenidos curriculares desde la óptica de lo que Nancy Fraser Ilama estrategias afirmativas y 
transformativas. Cuanto más se acerca a la segunda, más potencialidad tiene para modificar las relaciones simbólicas de dominación y de desigualdad que se encuentran soterradas en las nociones de justicia social de cada currículo.

He organizado el texto en tres grandes apartados. En el primero, expongo brevemente la noción de justicia social de Nancy Fraser y su definición de estrategias afirmativas y transformativas. En el segundo, describo cómo cada uno de los cuatro países define la relevancia y la pertinencia de los contenidos escolares y en especial de la enseñanza de la historia. Por último, me extiendo en el análisis de los contenidos curriculares dedicados a la conquista y colonización de América en educación obligatoria.

\section{Justicia social y educación}

Por economía expositiva, se puede decir que existen dos grandes corrientes de pensamiento que han vinculado la justicia social y la educación: la educación como redistribución y la educación como reconocimiento. Esta dicotomía se deriva de la oposición de las teorías de justicia social expuestas por Nancy Fraser (2006). De un lado tenemos lo que podemos Ilamar liberalismo social, que centra sus propuestas en el binomio libertad y equidad para una justa distribución de los bienes. Quizá sus más destacados representantes sean John Rawls, Amartya Sen y Martha Nussbaum. La educación, en cuanto un bien, estaría más vinculada a aspectos de ingreso y permanencia de los individuos dentro de su sistema y si este, bajo principios de equidad y de igualdad de oportunidades, es capaz de formar a los individuos con las capacidades de desempeño necesarias para desenvolverse en la sociedad actual. El conocimiento en esta corriente no se pone en duda, como lo muestran los grandes proyectos de políticas educativas que siguen este pensamiento, por ejemplo el Program for International Student Assessment (PISA) de la Organización para la Cooperación y Desarrollo Económico (OCDE).

Del otro lado, podemos ubicar las teorías de la justicia que consideran el reconocimiento de las diversas identidades como parte central de su propuesta. Destacados autores de esta corriente son Iris Marion Young y Alex Honneth, para quienes el derecho a la diferencia y al reconocimiento es la categoría moral cardinal (Fraser, 2006). Una intermedia, entre la visión redistributiva y las teorías del reconocimiento, es la propuesta de la propia Nancy Fraser. Esta autora considera que no hay justicia social si no hay redistribución (aspectos económicos), reconocimiento (temas culturales) y representación (ámbitos políticos) que permitan una paridad participativa de los diferentes individuos o grupos sociales (Fraser, 2006, 2008). Para alcanzar estos tres elementos, comúnmente se han tomado dos estrategias básicas: las de afirmación y las de transformación 
las estrategias afirmativas para reparar la injusticia intentan corregir los resultados desiguales de los acuerdos sociales sin tocar las estructuras sociales subyacentes que los generan. En cambio, las estrategias transformadoras aspiran a corregir los resultados injustos reestructurando, precisamente, el marco generador subyacente (Fraser, 2006, p. 72).

Es decir, mientras las primeras se centran en el resultado, las segundas buscan la transformación de la causas últimas.

El problema entonces es ¿cuándo un contenido curricular es potencialmente afirmativo o transformativo? La respuesta estaría en la capacidad de cada estrategia de ofrecer la posibilidad de reparar "la subordinación de estatus mediante la deconstrucción de las oposiciones simbólicas que subyacen a los patrones de valor institucionalizados en la actualidad" (Fraser, 2006, p. 73). Con base en lo anterior, analizo aquí los contenidos históricos curriculares a partir de los lentes de las estrategias afirmativas o transformativas. Cuanto más se aproximen a la segunda, es decir, cuanto más promuevan "la desinstitucionalización de los patrones de valor cultural que impiden la paridad de participación" (p. 71), mayor es la potencialidad de justicia social que poseen.

\section{Relevancia y pertinencia en educación}

La calidad educativa incluye, según cada país u organismo internacional, algunas de las siguientes dimensiones: eficacia, eficiencia, pertinencia, relevancia, equidad, flexibilidad, suficiencia o impacto, entre otras. Las cinco primeras son las más comunes. De esas me interesa resaltar dos, pues tienen las condiciones para ser observadas desde la perspectiva de las estrategias afirmativas y transformativas. Estas son pertinencia y relevancia, es decir, el para quién y el para qué de la inclusión de un determinado contenido en el currículo y sus formas de enseñanza y evaluación. La relación entre ambas es la siguiente:

La educación será relevante en la medida que promueva aprendizajes significativos desde el punto de vista de las exigencias sociales y del desarrollo personal, lo cual difícilmente ocurrirá si esta no es también pertinente; es decir, si no considera las diferencias para aprender que son fruto de las características y necesidades de cada persona (Laboratorio Latinoamericano de Evaluación de la Calidad de la Educación, 2008, p. 8).

El "para qué", o la relevancia de la educación, se fija a partir de la interpretación de lo que es el presente y lo que se desea para el futuro. Chile y México sostienen las finalidades educativas en políticas económicas, sociales y culturales de lo que se ha denominado comúnmente neoliberalismo, es decir, el proceso histórico iniciado en los años setenta del 
siglo pasado que tiene como principios insustituibles la desregularización del comercio, las privatizaciones de las industrias paraestatales y la reducción del gasto social. El principio de justicia social es la igualdad de oportunidades, por lo que las acciones educativas se centran en afirmar las condiciones de una equitativa competencia entre los individuos, lo que resultará en que cada individuo ocupará el lugar social que le corresponde según sus méritos (Dubet, 2010). Por tanto, la relevancia, en los países que sostienen en cierta medida el liberalismo social, radica en la afirmación de este tipo de sociedad, mientras que la pertinencia será un individuo (niño o joven) que se construye a sí mismo.

La actualización curricular del 2009 en Chile analiza el presente y mira el futuro en su definición de relevancia. Las exigencias

\begin{abstract}
de actualización, reorientación y enriquecimiento curriculares [...] se derivan de cambios acelerados en el conocimiento y en la sociedad, [por lo que se tiene] el propósito de ofrecer a alumnos y alumnas conocimientos, habilidades y actitudes, relevantes para su vida como personas, ciudadanos y trabajadores, así como para el desarrollo económico, social y político del país (Ministerio de Educación de Chile, 2009, p. 1).
\end{abstract}

En la lógica de evaluación la relevancia se limita a estándares de aprendizaje que miden la adquisición de "las competencias para la vida social, la vida individual y el desarrollo del país" (Consejo Asesor Presidencial para la Calidad de la Educación, 2006, pp. 90-91). La pertinencia, por su parte, tiene una marcada influencia psicológica ya que sigue al constructivismo cognitivo español y estadounidense de los años noventa que sostiene que el trabajo pedagógico

\footnotetext{
tiene por sujeto a los alumnos y alumnas, sus características, y sus conocimientos y experiencias previas. Centrar el trabajo pedagógico en el aprendizaje exige una enseñanza que desarrolla estrategias pedagógicas diferenciadas y adaptadas a los diversos niveles, ritmos y estilos de aprendizaje de los alumnos y alumnas (Ministerio de Educación de Chile, 2013a, p. 6).
}

México sigue el mismo camino de la economía desregulada y la psicología cognitiva, por lo que la relevancia educativa es parte de una estrategia afirmativa para la sociedad del conocimiento. El proyecto de articulación de la educación básica de 2011 considera que hay una demanda social por insertarse en la sociedad del conocimiento (Secretaría de Educación Pública, 2011a) y en el Sistema Nacional de Bachillerato $(\mathrm{SNB})$; lo relevante es

la formación de personas que puedan participar en la sociedad del conocimiento [con] sólidas bases formativas, [con] capacidad para 
aprender de forma autónoma a lo largo de la vida, y [con] habilidades para resolver problemas y desarrollar proyectos [que permitan abrir] la posibilidad de obtener ventajas en los mercados mundiales y nacionales (Secretaría de Educación Pública, 2008, p. 15).

La pertinencia por su parte, a diferencia de Chile, considera c on mucha más claridad aspectos individuales y culturales de los educandos, pues los procesos de enseñanza y aprendizaje deben responder a "las necesidades específicas de aprendizaje de cada uno de sus estudiantes, para que adquieran las competencias que permitan su desarrollo personal" (Secretaría de Educación Pública, 2011a, p. 11) y valorar, proteger y desarrollar las culturas y sus visiones y conocimientos del mundo a través de su inclusión en el currículo (p. 27).

Por su parte, los proyectos educativos descoloniales de Ecuador y Bolivia tienen una fuerte carga histórica, por lo que las finalidades educativas interpretan el presente a partir de las condiciones de sometimiento histórico de los campesinos, los indígenas y los afrodescendientes y su potencial liberación en las repúblicas plurinacionales actuales. Por esto la relevancia educativa tiene que ver con políticas económicas que han renacionalizado la riqueza del suelo y el subsuelo nacional, han regulado las relaciones comerciales, aumentado el gasto social, reducido la pobreza, mantenido -e incluso radicalizado- políticas democráticas y ampliado las políticas identitarias a lo intra- e intercultural. La justicia social se basa en el papel del Estado como garante de los derechos políticos, económicos, sociales y culturales y, aunque no niega la igualdad de oportunidades, en cierta medida se aproxima a lo que Francois Dubet llamó igualdad de posiciones, es decir, buscar que los lugares que se ocupan en la estructura social sean próximos entre sí (Dubet, 2010). Por tanto, la relevancia de la educación radica en su capacidad de desmontar los dispositivos culturales que producen la desigualdad, y la pertinencia se centra en las características culturales de las diferentes identidades comunitarias o étnicas.

La ley educativa boliviana expone su noción de relevancia en su primer artículo: la educación es

descolonizadora, liberadora, revolucionaria, anti-imperialista, despatriarcalizadora y transformadora de las estructuras económicas y sociales; orientada a la reafirmación cultural de las naciones y pueblos indígena originario campesinos, las comunidades interculturales y afrobolivianas en la construcción del Estado Plurinacional y el Vivir Bien (Ministerio de Educación del Estado Plurinacional de Bolivia, 2010, p. 4).

Para lograrlo, como muestra de lo imbricado que están los discursos educativos en la región, la educación también debe desarrollar "competencias, aptitudes y habilidades físicas e intelectuales que vincule la teoría 
con la práctica productiva; a la conservación y protección del medio ambiente, la biodiversidad y el territorio para el vivir bien" y contribuirá "al fortalecimiento de la unidad e identidad de todas y todos como parte del Estado Plurinacional" (Constitución Política del Estado Plurinacional de Bolivia, 2009). La pertinencia es culturalista, no psicologicista. La Ley Sañini-Pérez pretende que la educación desarrolle "programas educativos pertinentes a cada contexto sociocultural, lingüístico, histórico, ecológico y geográfico, sustentados en el currículo base de carácter intercultural" (Ministerio de Educación del Estado Plurinacional de Bolivia, 2010, p. 4) y se asegure "el acceso y permanencia a una educación adecuada en lo cultural y relevante en lo social, mediante políticas y procesos educativos pertinentes a las necesidades, expectativas e intereses de las personas, familias, comunidades y organizaciones" (Ministerio de Educación del Estado Plurinacional de Bolivia, 2010, p. 18).

En Ecuador la relevancia de la educación radica en su capacidad de centrarse en el ser humano y garantizar su desarrollo holístico; además de que es indispensable para "el conocimiento, el ejercicio de los derechos y la construcción de un país soberano" (Constitución de la República del Ecuador, 2008), debe responder al "interés público y no estará al servicio de intereses individuales y corporativos" y debe promover "el diálogo intercultural en sus múltiples dimensiones" (Constitución de la República del Ecuador, 2008). La pertinencia en el modelo ecuatoriano implica garantizar "a las y los estudiantes una formación que responda a las necesidades de su entorno social, natural y cultural en los ámbitos local, nacional y mundial" (Ley orgánica de educación intercultural, 2011, p. 11). Asimismo, el Estado debe "asegurar una educación con pertinencia cultural para los pueblos y nacionalidades, en su propia lengua y respetando sus derechos" (Ministerio de Educación de Ecuador, 2012, p. 57) por lo que hay que "transversalizar la interculturalidad para la construcción del Estado plurinacional y garantizar una educación con pertinencia cultural y lingüística para los pueblos afro ecuatorianos, montubios y para las nacionalidades y pueblos indígenas" (p. 76). La pertinencia está dada entonces por las condiciones sociales, culturales e históricas de los diferentes grupos que componen a las nacionales plurinacionales.

Aunque esquemática, la división entre proyectos educativos basados en el liberalismo social y los descoloniales muestra con claridad los conceptos de justicia social que subyacen tras las ideas sobre "para qué" y "a quién" educar. Chile y México, da continuidad a las reformas de los años noventa y sostienen su principio de pertinencia en el desarrollo psicogenético defendido por el constructivismo cognitivo de origen español (Plá, 2014) y su función es responder a la sociedad del conocimiento y a las relaciones económicas de libre mercado global, aunque en el caso mexicano no olvida los aspectos culturales y sociales de los educandos. Es 
una posición afirmativa y poco transformativa. Bolivia y Ecuador buscan la relevancia educativa en la transformación de toda relación de producción, material o simbólica, que reproduzca las añejas formas de dominación, mientras que la pertinencia se basa en las condiciones culturales de los diferentes grupos de cada país.

\section{Narraciones curriculares sobre la conquista de América}

Las estrategias afirmativas y transformativas en educación se pueden analizar a partir de los contenidos de ciencias sociales y específicamente de historia. La trama del relato histórico curricular puede reproducir las relaciones de poder y de estatus que subyacen en las estructuras sociales o, por el contrario, deconstruir esas mismas relaciones de poder para transformarlas y hacerlas más igualitarias. Un acontecimiento como la conquista y colonización de lo que hoy es parte importante de América Latina por el imperio español durante los siglos xv y xvı en el currículo escolar implica tomar posición sobre lo justificable o no de la dominación de un pueblo sobre otro; sobre ubicar cuándo se siembran las raíces de la identidad nacional; sobre el establecimiento de jerarquías entre conocimientos, culturas y procesos cognitivos con los que se deben pensar los pasados, justificar o rechazar el orden presente y aclarar la idea de inter-, multi- o pluriculturalidad que se prescribe, entre otros temas. En otras palabras, la enseñanza de acontecimientos históricos tan relevantes socializa patrones culturales que legitiman aspectos como las nociones de igualdad o desigualdad y por tanto de justicia social.

\section{Chile}

En los programas chilenos, actualizados en 2009 y de nuevo en 2012 y 2013, tras las presiones por romper con el sistema educativo pinochetista, por el proceso histórico del siglo xxı sudamericano que exigió un mejor y mayor reconocimiento a los pueblos indios y los estados plurinacionales y por dos décadas de "una nueva institucionalidad multicultural" (Smith, 2010) más relacionada con las políticas globales del reconocimiento a la diversidad que por su traducción en políticas públicas, la enseñanza de las ciencias sociales y la historia trataron de dar presencia al tema de la diversidad y por tanto de los pueblos originarios. Sin embargo, el tratamiento curricular es potencialmente una estrategia afirmativa, incluso reproduccionista. Parte de esto se debe al enfoque de enseñanza y parte a la narración curricular. Sobre el primero, la enseñanza de las ciencias sociales y de la historia en Chile tiene una perspectiva que imbrica la tradición disciplinar de enseñar a pensar históricamente surgida en Inglaterra 
durante la década de 1970 y continuada por diversos autores, como Sam Wineburg o Peter Lee entre muchos otros, y el currículo por competencias impulsado por proyectos como el Tuning y el Proyecto Definición y Selección de Competencias (DeSeCo) de la OCDE. De esta manera su relevancia, más allá de la formación ciudadana, radica en que enseñe a los estudiantes un conjunto de habilidades para que piensen la realidad presente y pasada y puedan al mismo tiempo enfrentar al mundo cambiante de la actualidad (Ministerio de Educación de Chile, 2009, pp. 195-197). Es un enfoque afirmativo en cuanto ofrece condiciones aparentemente objetivas para desenvolverse en el mundo laboral flexible del mercado global. No modifica el modelo económico y social injusto, más bien lo reafirma al dar elementos (habilidades cognitivas) para sobrevivir en él.

La narración curricular de la conquista y colonización, así como el lugar que ocupan los pueblos indígenas en la historia chilena, continúa con la lógica afirmativa. Esto se hace en tres aspectos: el binomio unidad/diversidad del mestizaje; la conquista dentro de la historia universal; y la subalteridad de los pueblos indios. El primer caso es común en América Latina, sobre todo a partir de mediados del siglo xx, cuando las identidades criollas pierden fuerza en el currículo escolar y se hace hegemónica la idea de un sincretismo cultural basado en la unión racial. Los pueblos originarios aparecen en primer y segundo grados de primaria desde una perspectiva etnográfica que los reduce a mercancía para el consumo del turismo cultural (Ministerio de Educación de Chile, 2011a) y a las etapas primitivas del desarrollo histórico. La trama narrativa es la siguiente:

En segundo básico se familiariza a los alumnos con la diversidad cultural de la sociedad chilena [...]. En primer lugar, se estudian los pueblos indígenas que habitaron el actual territorio nacional en el período precolombino, enfatizando en el reconocimiento de su legado en expresiones del patrimonio cultural y en su presencia en la actualidad. Luego se abordan los aportes realizados por españoles e indígenas y la importancia del mestizaje en la conformación de nuestra sociedad. Finalmente, se destacan los aportes realizados por inmigrantes de diferentes naciones a lo largo del tiempo (Ministerio de Educación de Chile, 2013b, p. 36).

Este esqueleto narrativo se sostiene en la relación binaria unidad/ diversidad en la que la pluralidad funciona en la medida en que representa la unidad de la identidad chilena. A pesar de que son muchas culturas, no se conforman identidades chilenas sino la identidad chilena. Un ejemplo gráfico es la imagen con la que concluye un material didáctico del Ministerio de Educación, donde un conquistador y una indígena (más pequeña que el español) carga al fruto de la unión: el niño mestizo (Olivares, s. f.). 
El segundo elemento es central para comprender el carácter afirmativo del currículo chileno. A Historia Occidental, medida desde la que se determina el nivel de progreso de un pueblo, se le dedican tres cursos completos: séptimo y octavo básico y primero medio. La estructura evolucionista - del estado de naturaleza a la civilización- y dual -occidente/ no-occidente, civilizado/salvaje; moderno/tradicional (Quijano, 2000)sigue los parámetros eurocéntricos decimonónicos. En esta lógica se encuentra la conquista de América. En octavo grado el Aprendizaje Esperado número 03 es muy claro: hay que reconocer el impacto del descubrimiento de América en Europa y en América tomando en cuenta, entre otros aspectos, la ampliación del mundo conocido, la discusión de la naturaleza humana de los indígenas y la incorporación de América a la cultura occidental. Los pueblos originarios, como la raíz más antigua de lo chileno, se pierde completamente: los americanos como los mapuche no son capaces de conocer su propio territorio por lo que tienen que ser descubiertos, también los occidentales, entre los que se encuentran los propios chilenos, tienen que reflexionar sobre la naturaleza humana del indio y finalmente, para que todo eso sea posible, se niega todo lo prehispánico para convertirse en parte del hemisferio occidental.

Lo anterior genera, como afirma Andrea Riedemann, un proceso de alteridad al ubicar a los mapuche como el otro interior y de subalternidad al "hablar de ellos" (Riedemann, 2012, p. 202), aunque a mi parecer no se queda ahí, pues la subalternidad como lo ha expuesto Gayatri Spivak también implica "hablar por ellos" (Spivak, 2009; Smith, 2010). El ejemplo más claro no se encuentra en Historia Universal, sino en Historia Chilena de quinto grado, cuando se sugiere en el portal del Currículum en línea, una lectura titulada "La voz de los indígenas", que es un fragmento de un sermón del fraile dominico Antonio Montesino en la isla de la Española en 1511, en el que despotrica por la violencia de los encomenderos contra los indígenas caribeños. A pesar de que los procesos de alteridad y subalternidad se mantienen en los programas de Historia Chilena, es necesario reconocer que para la educación media hay un intento tímido por abrir la interpretación del conflicto mapuche en sus consecuencias culturales y económicas, así como la proyección de "la situación de los mapuches en el Chile de hoy" (Consejo Nacional para la Cultura y las Artes, 2011, p. 35).

Visto lo anterior desde los lentes de la relevancia y la pertinencia educativas, las acciones transformativas para modificar las oposiciones binarias que establecen jerarquías raciales y culturales parecen estar todavía lejos de alcanzarse. La relevancia en la Ley General de Educación chilena se centra en el desarrollo pleno de la persona (entendido como desarrollo de competencias), en un marco de identidad nacional y multiculturalidad (Ministerio de Educación de Chile, 2012), a lo que la educación histórica trata de darle respuesta. Sin embargo, vimos que el multiculturalismo en las 
narraciones históricas curriculares más bien reproduce la homogeneidad mestiza, ubicando a los pueblos indios en posición de subalternidad y por tanto de imposibilidad de paridad participativa. En cuanto a la pertinencia, basada en el pensamiento científico y las habilidades de pensamiento, se puede ver cómo en realidad reproduce la subalternidad de los pueblos indios al proponer fuentes que en realidad ocultan la voz indígena.

\section{México}

Los programas de estudio en México para la educación básica y obligatoria llevan veinticinco años en reformas permanentes. A lo largo de estas dos décadas y media los pueblos indígenas fueron ganando peso en los programas de estudio, fundamentalmente por el movimiento del Ejército Zapatista de Liberación Nacional (EZLN), que desde 1994 tiene una presencia central en el tema indígena, pero también por el largo proceso de transición del sistema educativo basado en una identidad homogénea y centralizada, a imagen del sistema francés, hacia la idea cada vez más descentralizada y con base en las políticas de identidad de corte anglosajón. El resultado es que el Acuerdo 592 de 2011 que define el currículo considera dentro de la relevancia la atención a la diversidad, con especial énfasis en las culturas indígenas. A pesar de que este reconocimiento es parcialmente transformativo, la exclusión cognitiva y las estructuras narrativas de los currículos históricos escolares son una estrategia afirmativa.

La exclusión cognitiva se puede observar en el enfoque de la enseñanza de la historia y en general en toda la Educación Básica y la Educación Media Superior. Las competencias son parte de un proceso educativo que tiende a simplificar el conocimiento en una sola forma de pensamiento genérico (Young, M. 2008). Esto produce creaciones didácticas o curriculares como las competencias disciplinares, es decir, "Ios conocimientos, habilidades y actitudes asociados con las disciplinas en las que tradicionalmente se ha organizado el saber" (Secretaría de Educación Pública, 2008). En historia son tres: tiempo y espacio histórico, manejo de información histórica y conciencia histórica para la convivencia (Secretaría de Educación Pública, 2011a). Según se argumenta, en concordancia con el caso chileno, son habilidades universales del pensar históricamente que pueden traducirse con facilidad en estándares curriculares para su evaluación. Pero el pensamiento histórico entendido así es un pensamiento occidental (alemán en un inicio) que se expandió en las universidades del mundo entero de la mano del imperialismo británico y francés del siglo xIx. Como tal, ha negado múltiples formas de pensar la historia que no provengan de esa fuente primigenia. Las competencias son una nueva etapa de esta expansión cognitiva, que niega la posibilidad de pensar la historia de otra manera. El tema es muy complejo y no tengo espacio aquí para profundizar en él, por lo que solo alcanzo a resumirlo así: en México la población 
indígena es obligada a pensar la historia del país en términos occidentales, y a la población no indígena se le impide conocer los pluriversos cognitivos (Carrillo, 2006) que existen allí para pensar el pasado. Por tanto, no son pertinentes a la diversidad cultural mexicana ni son relevantes para desarrollar la interculturalidad.

La narración histórica curricular, como en el caso chileno, puede verse a través del binomio unidad/diversidad del mestizaje; lo mesoamericano y la conquista dentro de la historia universal; y la subalternidad de los pueblos indios. Sobre la unidad/diversidad México tiene un ya viejo discurso de identidad mestiza, surgido del proyecto de unidad nacional de mediados del siglo xx que saldó la disputa histórica del origen mexicano en la simbiosis cultural, de base racial, denominada mestizo, en el que los libros de texto de historia fueron fundamentales (Vásquez, 2000) y que está muy sedimentado en la historia escolar actual (Plá, 2014). El resultado es la unidad producida por la diversidad, es decir, las múltiples identidades forman una única identidad mexicana, de ahí que se trabaje la conquista de México, oculta entre dos periodos históricos, para fomentar "una reflexión [por parte de los alumnos] sobre el mundo prehispánico para comprender que este y la conformación de Nueva España constituyen un periodo fundacional" (Secretaría de Educación Pública, 2011b, p. 28).

Si se argumenta que México se funda con la conquista y la colonización es para ubicarlo en la historia de la globalización. Esto genera un proceso doble, por un lado rompe en parte con el eurocentrismo, pero por otro construye una historia armoniosa de integración de los mercados en la que México es actor trascendental. El siglo xvı es la primera expresión del mundo globalizado y el aprendizaje esperado es que el alumno describa "el proceso de integración económica en el siglo xvı" (Secretaría de Educación Pública, 2011a, p. 570) donde el proceso se dio gracias al intercambio económico y cultural cuasi consensuado por ambas partes. Lo mismo sucede cuando se trabaja la llegada de Cristóbal Colón a América como "Encuentro de América y Europa" (p. 409) y se mencionan las consecuencias de la conquista sin aclararlas y mucho menos discutir las implicaciones de la dominación de un pueblo sobre otro. La historia edulcorada llega quizá a su clímax con la equivalencia entre españoles y africanos bajo el título de inmigrantes (Secretaría de Educación Pública, 2011a, p. 507). A pesar de esto, hay que reconocer que los contenidos de Historia Universal sí amplían notablemente la mirada a otras partes del mundo, mucho más allá de Occidente y más que el caso boliviano, como veremos.

A pesar de estas inclusiones, el racismo en el sistema educativo mexicano y en la enseñanza de la historia es añejo, abarca a sectores mucho más amplios que las comunidades indígenas y no ha sido lineal a lo largo de la historia (Rodríguez y Velasco, 2014). Por ejemplo, en el 
estudio de Dolores Ballesteros se demuestra cómo el impulso del movimiento zapatista y las políticas internacionales por el reconocimiento a la multiculturalidad provocaron que los contenidos curriculares para educación secundaria incluyeran más temas sobre los indígenas y los afromexicanos, pero también demuestra cómo en 2011 hubo una nueva disminución del tema. Asimismo sus formas de representación en los libros de texto de secundaria (Ballesteros, 2015) y de primaria (Pacheco y Navarro, 2011) siguen siendo excluyentes, en la medida en que solo ven la diversidad con relación al mito fundacional mestizo y se ubican en la subalternidad, pues poco o nada se les da voz a los grupos marginados (Young, K., 2010).

La subalternidad, la oposición binaria unidad/diversidad -que puede traducirse mestizo/indígena- y la historia edulcorada de la integración económica mundial son similares a la estructura chilena, con la diferencia de que lo occidental es ocupado en México por la globalización o la integración de México al mercado mundial. Por estas razones es claro que aunque los programas de historia promueven una reducción del eurocentrismo y que incluso la Constitución Política reconoce la pluralidad cultural, todavía el relato histórico del currículo promueve acciones afirmativas que no favorecen la paridad participativa desde el derecho a la diferencia en la sociedad, pues la relevancia del contenido no está en el reconocimiento de la diversidad, sino en la explicación de la integración económica y del conjunto de habilidades cognitivas que permiten tener éxito en él.

\section{Ecuador}

El currículo ecuatoriano es muy distinto al mexicano y al chileno en algunos aspectos, pero en otros es extrañamente similar. Al analizar los contenidos históricos y el enfoque de su enseñanza da la impresión de que las interpretaciones históricas tuvieran un impulso descolonial y transformativo mientras que el diseño pedagógico mantuviera el carácter afirmativo del liberalismo social. Por ejemplo, los estudios sociales dividen claramente Geografía e Historia como las dos asignaturas ejes del campo, mientras que Formación Ciudadana es la dimensión valorativa de estos contenidos (Ministerio de Educación de Ecuador, 2010, p. 78). A su vez, la Historia del Ecuador sigue puntualmente el libro coordinado por el historiador Enrique Ayala, Nueva historia del Ecuador, que desde su primera edición en 1993 valoró la importancia de incluir a los indígenas y a los afroecuatorianos como actores fundamentales de la historia de esta república sudamericana. En otras palabras, hay una intención deliberada de romper con la historia fragmentada de los pueblos indios que caracterizó la identidad mestiza del siglo xx, como se puede leer en el libro Estudios sociales de sexto grado:

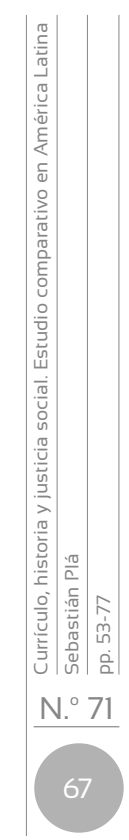


desde la conquista, los pueblos originarios ya no fueron tomados en cuenta en nuestra historia tradicional. Pero los indígenas han sido parte fundamental de nuestro pasado común. Siguen presentes hasta ahora en nuestra vida como país y son actores importantes de la sociedad (Ayala, 2011, p. 41).

La narración curricular de la conquista de Ecuador y de la época colonial se interpreta como

uno de los temas más polémicos de la Historiografía Latinoamericana. Su estudio se aborda a partir de presentar a la España de la conquista y a los conquistadores frente a los pueblos indígenas que fueron sojuzgados y ofrecieron diversas formas de resistencia a la invasión y al coloniaje. Se desechan, de este modo, visiones unilaterales o racistas que pintan a los aventureros españoles como superhéroes y a los indígenas como inferiores; pero también se ponen de lado explicaciones simples y etnocentristas que no dan cuenta de la complejidad del proceso[...],

Un elemento muy importante es el estudio de la permanencia de las comunidades indígenas, junto con la irrupción de los blancos y la traída de los negros desde África, lo cual dio origen al mestizaje, cuya presencia en la historia no debe ser entendida como una mezcla racial sino como una nueva realidad cultural (Ministerio de Educación de Ecuador, 2010, p. 93).

Cuatro aspectos importantes de la cita (reconocimiento del derecho a la diversidad dentro de la identidad; lo polémico de la interpretación histórica, la lucha contra las interpretaciones racistas y etnocéntricas y el mestizaje como cultural y no racial) pueden pensarse en su conjunto como un explícito uso político de la historia en la escuela, a diferencia de México y Chile que lo ocultan bajo palabras científicas. Afirmar que los "indígenas fueron sojuzgados" al igual que aseverar que los criollos fueron parte de la dominación de los indígenas o hacer que los estudiantes describan "el desplazamiento forzoso de grandes poblaciones negras desde África a América" (Ministerio de Educación de Ecuador, 2010, p. 88) son ejemplos del posicionamiento político del programa escolar. Incluso se sostiene que el estudio de

la conquista y colonización americana no puede ser neutro. Debe ser la base de un rechazo de la violencia y la imposición como forma de relación entre las personas y entre los pueblos. Por otra parte, debe dar pie para valorar la gran diversidad de las culturas americanas (indígenas, mestizas, afro), que son nuestra riqueza y clave para nuestro presente y futuro (Ministerio de Educación de Ecuador, 2011, p. 51).

Exponer las condiciones de sojuzgamiento, explotación y en buena medida genocidio de los pueblos indios (y africanos) durante los procesos de colonización del siglo xvı es enseñar la verdad histórica con una 
intención de reconocer la pluralidad nacional y las posiciones de poder desde las que se han establecido las relaciones entre los diferentes grupos de una sociedad. Deconstruir la dimensión histórica de desigualdad de estatus, como señala Fraser, es un paso importante para alcanzar la justicia social en América Latina.

Pero el programa de historia en el sistema educativo ecuatoriano también presenta paradojas e incluso contradicciones. Por ejemplo, se denomina a la llegada de Cristóbal Colón a Guanahani en 1492 como "Descubrimiento de América" y no duda en dividir el planeta anterior a esa fecha como Viejo y Nuevo Mundo. Esta contradicción trata de salvarse en el libro de texto de sexto grado: "Ilamarlo 'descubrimiento' es ver la realidad solo desde el punto de vista de los europeos, que consideraron al Nuevo Mundo como "tierra de conquista" y a sus habitantes como objetos de explotación" (Ayala, 2011, p. 41). Esta forma de narrar la historia es un intento por desdoblar los conceptos occidentales y vencer así el eurocentrismo para comenzar un proceso de descolonización simbólica. Sin embargo la lucha contra la historia eurocéntrica se da en otros ámbitos de la narración histórica curricular. En los dos últimos grados de educación obligatoria se enseña historia mundial y el Viejo Mundo es amplio: la historia comienza con la diseminación de la humanidad desde África, se estudia Latinoamérica e incluso se mencionan en el mismo nivel la independencia de Estados Unidos, la Revolución Francesa y la Independencia de Haití; este último es un acontecimiento fundamental en la interpretación de la historia decolonial (Mignolo, 2003; Quijano, 2000).

No cabe lugar a dudas de que tras casi dos siglos de dominio criollo y mestizo de la historia escolar ecuatoriana, el proyecto curricular de 2010 tiene la intención deliberada de responder a la relevancia desde la necesidad de construir una identidad diversa, y a la pertinencia desde la diversidad que caracteriza a la población que habita en Ecuador. Aunque al igual que México y Chile utiliza la dicotomía unidad/diversidad, esta última no construye una teleología mestiza y racial que unifica a la identidad, por el contrario elimina el carácter racial de lo mestizo y promueve la unidad desde la diversidad, es decir, el reconocimiento a las identidades ecuatorianas. Esta nueva forma de narrar el pasado y en especial la conquista y la colonización puede considerarse una acción transformativa que puede llegar a producir a largo plazo un cambio simbólico que promueva la justicia social. Sin embargo, las paradojas del currículo ecuatoriano superan los relatos del pasado nacional, pues son contradicciones pedagógicas más amplias. Cada uno de los contenidos que hemos trabajado aquí son "Destrezas con criterios de desempeño", es decir, la destreza como "la expresión del 'saber hacer' en los estudiantes, que caracteriza el dominio de la acción" y los criterios de desempeño como la orientación y la precisión del "nivel de complejidad en el que se debe realizar la acción,

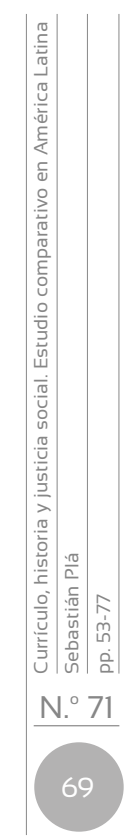


según condicionantes de rigor científico-cultural, espaciales, temporales, de motricidad, entre otros" (Ministerio de Educación de Ecuador, 2011, p. 11). Descrito así parece un currículo por competencias heredado de las reformas curriculares neoliberales de finales del siglo pasado. Las nuevas interpretaciones históricas descoloniales se colocan dentro de un molde global de competencias, destrezas o criterios de evaluación como si lo pedagógico y el diseño curricular en su conjunto no fueran tan políticos y necesitados de deconstrucción como las interpretaciones del pasado. Un molde afirmativo con contenido transformativo.

\section{Bolivia}

Bolivia, al igual que Ecuador, ha reconstruido su narración histórica curricular, pero sin montarse en las reformas de los años noventa del siglo xx. Utilizan poco los conceptos de competencias, destrezas o criterios de evaluación. Incluso en la primera etapa del proyecto se rechazó el término calidad de la educación (Yapu, 2010). Han resignificado el diseño curricular desde parámetros culturales propios, es decir, guiados por los principios de lo que denominan pedagogía intercultural e intracultural, tanto en el diseño curricular como en los contenidos históricos. Rompen con el dualismo diversidad/unidad mestiza, fracturan la historia eurocéntrica y definitivamente subvierten el orden jerárquico que ubicaba a grandes sectores de la población en el lugar de subalternos. Esto se ve desde la piedra angular del proyecto: la función de la educación es descolonizar. Es como si todo el sistema educativo estuviera estructurado para deconstruir las relaciones binarias de poder epistemológico de saber universal/saber local y saber científico/saber tradicional o las relaciones binarias identitarias de occidente/no occidente y moderno/tradicional heredadas del periodo de coloniaje y de etapas posteriores. Es, en términos de la teoría decolonial latinoamericana, un esfuerzo por deconstruir la colonialidad del poder o la racionalidad occidental y racista que permaneció en Bolivia después de obtener la independencia boliviana del dominio español. Por lo anterior se puede afirmar que los programas de estudio bolivianos forman parte de una estrategia potencialmente transformativa por la justicia social, lo que no implica ciertos riesgos o la presencia de miradas críticas del proceso.

La dimensión histórica de los programas se ve claramente en los contenidos del área de ciencias sociales para los seis años de la Educación Secundaria Comunitaria Productiva. En los programas de estudio de ciencias sociales dedicados a temas del pasado, aunque se incluyen contenidos de historia internacional y en especial latinoamericana, es básicamente una historia de los pueblos originarios, como lo son desde otros lugares epistemológicos, el currículo francés o el japonés. En un apretado resumen, se puede decir que se inicia con los referentes históricos y religiosos del 
Abya Yala para seguir con la invasión europea, la implantación violenta de la religión, la imposición cultural, la resistencia de los pueblos originarios durante el periodo colonial y las desigualdades económicas, políticas y educativas que produjo. Bolivia independiente sigue viéndose como periodo de dominación bajo el término neocolonial. En Historia Universal, se trabajan brevemente algunas ideas de la llustración, pero sobre todo el imperialismo norteamericano y el papel actual de los organismos internacionales (el FMI, el BM, la FAO, entre otros), en la imposición del neoliberalismo. Dan también mucho espacio a la revolución nacional de 1952 (reforma agraria, procesos de nacionalización y voto universal para indígenas y campesinos), la defensa del agua en 2000 y del gas en 2003. Por último, retomando lo estudiado a lo largo de toda la Educación Secundaria, se concluye con el Estado Plurinacional actual.

Las interpretaciones históricas de la conquista y el periodo colonial siguen el mismo camino que los objetivos específicos $y$, a diferencia de Chile y México, consideran que el colonialismo, sobre todo la colonialidad (permanencia de las relaciones de poder raciales y epistemológicas establecidas desde la Colonia hasta la actualidad) es un proceso de larga duración que debe ser combatido en el presente. Para esto los contenidos históricos dan continuidad a la historia de los pueblos indios, evitando su fragmentación narrativa o su exclusión de los procesos nacionales de los últimos doscientos años. También describen la Colonia desde el ámbito económico, por ejemplo al prescribir el estudio de la explotación de la fuerza laboral en la Colonia y en la República, denunciando las formas de sometimiento ejercidas contra los pueblos originarios y el saqueo y la enajenación de los recursos y derechos de la Madre Tierra (Campo de saberes y conocimientos. Comunidad y sociedad. Educación secundaria comunitaria productiva, 2011, p. 85). Otro espacio curricular para las interpretaciones históricas se da en las orientaciones metodológicas al profesor, donde se le sugiere la "organización de debates colectivos para analizar sobre el mayor genocidio que se dio en esta parte del mundo" (p. 84).

Pero la potencia del relato histórico curricular boliviano no solo es la ilación de los acontecimientos y procesos históricos, sino su función social y su consiguiente interpretación. Los objetivos específicos para la enseñanza de estos contenidos no son aprendizajes esperados o destrezas con criterios de desempeño sino la valoración crítica del pasado que "deconstruye la historia [colonial y de colonialidad] para reconstruir la historia de los pueblos" y legitimarla (Campo de saberes y conocimientos. Comunidad y sociedad. Educación secundaria comunitaria productiva, 2011, pp. 92-93). Además, el trabajo con contenidos históricos tiene una impronta transformativa: estos promueven "la transformación de realidades desiguales", y consolida la unidad nacional, la "defensa de la integridad cultural" y "el proceso de descolonización y despatriarcalización del 
Estado Plurinacional" (Campo de saberes y conocimientos. Comunidad y sociedad. Educación secundaria comunitaria productiva, 2011).

Las críticas al proyecto educativo del Estado Plurinacional de Bolivia son significativas y variadas. Una ha sido la esgrimida por Carmen Osuna (2013), quien sostiene que la construcción entre lo propio y ajeno del proyecto curricular intracultural, intercultural y plurilingüe corre el riesgo de debilitar su intención descolonizadora al presentar ambigüedades en la definición de lo propio, lo que termina por producir estereotipos maniqueos que niegan transformaciones constantes y dinámicas de las identidades de los pueblos originarios (Osuna, 2013, p. 465). Una segunda crítica, o mejor advertencia, está en la reproducción del modo de educación "bancario" occidental, es decir, la subordinación de los educandos al aprendizaje de contenidos planificados, pero ahora ya no con saberes europeos sino con los propios saberes indígenas. "Llenar cualquier currículo con contenidos indígenas no tiene ninguna relevancia, si la pedagogía y didáctica que se usan para enseñar dichos contenidos continúa educando niños y niñas para que no sean avas guaraníes, ni runas quechuas, ni jaqis aymaras" (Quintanilla Coro, 2012, p. 190). Por último, con relación a los contenidos históricos, quiero agregar que la legitimación de la historia de los pueblos originarios sin mirar a otras partes del mundo corre el riesgo de establecer narraciones históricas incapaces de ver lo intercultural por dos razones: la primera es interna y radica en que se llegue a la tiranía de la mayoría, en la que el derecho a la diversidad no indígena sea excluido de los relatos históricos y por tanto no tenga espacio en la historia escolar. La segunda es externa y tiene que ver con el etnocentrismo excesivo que presentan los temas históricos, en los cuales la historia de otros pueblos (africanos, asiáticos, europeos, americanos y oceánicos) queda fuera de los contenidos de aprendizaje. A pesar de estas justificadas críticas, vistos los contenidos históricos del proyecto educativo boliviano desde la relevancia (la posibilidad de construir nuevas relaciones sociales que rechacen la colonialidad epistemológica y racial) y la pertinencia (responder a los contextos culturales e identitarios de los educandos), no hay duda de su potencial transformativo para cambiar la desigual e injusta relación simbólica que ha predominado en el país durante siglos.

\section{Currículo, historia y justicia social}

La relación entre los contenidos históricos, el diseño curricular y la justicia social muestran el papel otorgado a la educación, sea esta para la transformación de las relaciones simbólicas de poder a partir del reconocimiento de la diferencia, o de la adaptación de los estudiantes a estructuras de dominación preestablecidas. A lo largo del texto hemos visto 
cómo en los casos chileno y mexicano, en la enseñanza de la historia la relevancia reconoce el derecho a la diversidad en la medida en que no cuestiona al sistema social, y económico, predominante. No sucede lo mismo en los casos boliviano y ecuatoriano, en los que la relevancia y la pertinencia de los contenidos son parte de una estrategia transformativa de las relaciones de poder heredadas desde la Colonia. Pero también hemos visto que entre ambos grupos (el liberalismo social y el descolonial) existen diferencias internas, pues México otorga peso a la interculturalidad mientras que Ecuador da continuidad a la lógica curricular basada en habilidades cognitivas de corte disciplinar.

Lo anterior implica que, en cierta medida, si observamos las lógicas curriculares y las formas de enseñanza prescritas por los diferentes programas de estudio, se puede concluir que Chile, México y Ecuador siguen principios heredados por la teoría del capital humano (en forma de competencias) y el constructivismo cognitivo (representado por habilidades). En el caso ecuatoriano, es una inconsistencia pedagógica significativa, pues no se considera al diseño curricular como espacio de lucha política, pero sobre todo no se reconocen las diversidades epistemológicas dentro de los programas de estudio, lo cual es contrario al carácter plurinacional del país. En cambio, en Bolivia las propuestas pedagógicas dan continuidad al proyecto descolonial y reconocen los diversos saberes, incluida la historiografía profesional, como formas legítimas de construcción de significados sobre el pasado dentro de la escuela. Esta hace que en lo pedagógico solo Bolivia proponga una estrategia transformativa que rompa con la oposición binaria ciencia/universalismo por un lado, saberes/localismo por el otro.

Por último, los países categorizados con el término liberalismo social estructuran sus narraciones históricas escolares con la lógica afirmativa, es decir, reafirman las condiciones objetivas y simbólicas de desigualdad a partir de tres elementos: niegan el derecho de los pueblos indios a hablar dentro de las interpretaciones históricas, no rompen completamente con el eurocentrismo narrativo y por tanto continúan legitimando esa perspectiva como forma de pensamiento histórico y, por último, dan continuidad a la idea de identidad nacional basada en una raza mestiza, que niega la pluralidad. En cambio los países descoloniales trocan la perspectiva de los acontecimientos históricos para mirarlos desde los grupos tradicionalmente marginados, reconocen las identidades plurales y de ahí su carácter plurinacional, y tratan de dar voz a los grupos históricamente sojuzgados, como los indígenas y los afrodescendientes. Con base en esto, se puede suponer que ambos países, más Bolivia que Ecuador, han diseñado en el ámbito educativo estrategias transformativas con el potencial de deconstruir las relaciones simbólicas y de poder que impiden un reconocimiento y una representación adecuada en una 
sociedad que busca la paridad participativa como elemento central de la justicia social. Esto no implica que no habrá riesgos en el futuro, pero con los magros resultados de los sistemas educativos latinoamericanos en la consecución de la justicia social, considero que por lo menos debemos darles el beneficio de la duda. 


\section{Referencias bibliográficas}

Ayala Mora, E. (2011). Estudios sociales 6. De acuerdo al nuevo currículo de la Educación General Básica. Quito: Corporación Editora Nacional.

Ballesteros Páez, M. (2015). Race and racism in Mexican history textbooks: A silent presence. En M. Araújo y S. Maeso. Eurocentrism, Racism and Knowledge (pp. 209-228). Hampshire: Palgrave Macmillan.

Consejo Nacional para la Cultura y las Artes. (2011). Conociendo a la cultura Mapuche. Guía de diálogo intercultural para el turismo indígena. Valparaíso, Chile.

Constitución de la República del Ecuador. (2008). Quito, Ecuador. CarriIlo Trueba, C. (2006). Pluriverso. Un ensayo sobre el conocimiento indígena contemporáneo. Ciudad de México: Universidad Nacional Autónoma de México.

Constitución política del Estado Plurinacional de Bolivia. (2009). Congreso Nacional. La Paz, Bolivia.

Dubet, F. (2010). Repensar la justicia social: contra el mito de la igualdad de oportunidades. Buenos Aires: Siglo Veintiuno.

Fraser, N. (2006). La justicia social en la era de la política de la identidad: redistribución, reconocimiento y participación. En N. Fraser y A. Honneth. ¿Redistribución o conocimiento?: un debate político-filosófico (pp. 17-88). Madrid: Morata.

Fraser, N. (2008). Escalas de justicia. Barcelona: Editorial Herder.

Goodson, I. F. (1995). Historia del currículum: la construcción social de las disciplinas escolares. Barcelona: Pomares-Corredor.

Laboratorio Latinoamericano de Evaluación de la Calidad de la Educación. (2008). Reflexiones en torno a la evaluación de la calidad educativa en América Latina y el Caribe. Santiago de Chile: Autor.

Legardez, A. (2003). L'Enseignement des Questions sociales et historiques, socialement vives. Le cartable de Clio, 3.

Ley orgánica de educación intercultural. (2011). Asamblea Nacional. Quito, Ecuador.

Mignolo, W. (2003). Historias locales/diseños globales: colonialidad, conocimientos subalternos y pensamiento fronterizo. Madrid: Akal.

Ministerio de Educación de Chile. (2009). Objetivos fundamentales y contenidos mínimos obligatorios de la Educación Básica y Media. Actualización 2009. Santiago de Chile, Chile.

Ministerio de Educación de Chile. (2011). Historia, Geografía y Ciencias Sociales. Programa de estudio. Segundo año medio. Santiago de Chile, Chile. 
Ministerio de Educación de Chile. (2012). Historia, Geografía y Ciencias Sociales. Educación Básica. Bases Curriculares. Santiago de Chile, Ministerio de Educación.

Ministerio de Educación de Chile. (2013a). Decreto 254. Objetivos fundamentales y contenidos mínimos obligatorios de la Media. Ministerio de Educación. Santiago de Chile, Chile: Autor.

Ministerio de Educación de Chile. (2013b). Historia, Geografía y Ciencias Sociales. Programa de estudio. Segundo año básico. Chile, Santiago de Chile.

Ministerio de Educación de Ecuador. (2010). Actualización y fortalecimiento curricular de la Educación General Básica 2010. 6. ${ }^{\circ}$ año. Área de Ciencias Sociales. Quito, Ecuador: Autor.

Ministerio de Educación de Ecuador. (2011). Actualización y fortalecimiento curricular de la Educación General Básica 2010. 8. ․ 9. y 10. ${ }^{\circ}$ años. Área de Ciencias Sociales. Quito, Ecuador.

Ministerio de Educación de Ecuador. (2012). Marco legal educativo. Quito, Ecuador: Autor.

Ministerio de Educación del Estado Plurinacional de Bolivia. (2010). Ley de la educación "Avelino Siñani-Elizardo Pérez". N. ${ }^{\circ}$ 70. La Paz, Bolivia.

Ministerio de Educación del Estado Plurinacional de Bolivia. Viceministerio de Educación Regular. (2011). Campo de saberes y conocimientos. Comunidad y sociedad. Educación secundaria comunitaria productiva. La Paz, Bolivia.

Olivares, C. (s. f.). Pueblos originarios de Chile. Santiago de Chile: Currículo en línea. Recuperado de: www.currículumenlineamineduc.cl/605/ w3-propertyvalue-57655.htm

Osuna, C. (2013). Educación intercultural y revolución educativa en Bolivia. Un análisis de proceso de (re)esencialización cultural. Revista Española de Antropología Americana, vol. 43; 2, 451-470.

Pacheco Ladrón de Guevara, L.; Navarro Hernández, M. y Cayeros López, L. (2011). Los pueblos indios en los libros de texto gratuitos. Revista Mexicana de Investigación Educativa, 49, 525-544.

Pinar, W. (2004). What is Currículum Theory? Mahwah, N. J.: Lawrence Erlbaum.

Plá, S. (2014). Mestizos e inconclusos. Interpretaciones sobre la historia de México en Bachillerato. Revista Mexicana de Investigación Educativa, 61, 483-509.

Quijano, A. (2000). Colonialidad del poder, eurocentrismo y América Latina. En E. Lander (comp.). La colonialidad del saber: eurocentrismo y ciencias sociales. Perspectivas Latinoamericanas. Buenos Aires: Clacso.

Quintanilla Coro, V. H. (2012). La perspectiva de descolonización educativa intra-cultural e intercultural. Estudios Bolivianos, 17, 187-219. 
Riedemann, A. (2012). Representaciones cambiantes e historia fragmentada: Ios Mapuche en los textos escolares de historia de Chile, 18462000. Tesis doctoral. Berlín: Freie Universität.

Rodríguez Ledesma, X. y Velasco Cruz, S. (2014). Otredad y construcción de futuro. La educación para los indígenas en México, un balance histórico. Revista Española de Educación Comparada, 23.

Santisteban, A. (2010). La formación de competencias de pensamiento histórico. Clío \& Asociados, 14, 34-56.

Secretaría de Educación Pública. (2008). Acuerdo Secretarial 442. Ciudad de México.

Secretaría de Educación Pública. (2011a). Acuerdo Secretarial 592. Ciudad de México.

Secretaría de Educación Pública. (2011b). Programas de estudio 2011. Guía para el maestro. Educación Básica. Secundaria. Historia. Ciudad de México.

Smith, S. (2010). Manuales escolares de Historia y Ciencias Sociales y subalternidad mapuche en un Chile multicultural. Tinkuy, 12, 53-72.

Spivak, G. C. (2009). ¿Pueden hablar los subalternos? Barcelona: MNAC, Museu d'Art Contemporani de Barcelona.

Vázquez, J. (2000). Nacionalismo y educación en México. Ciudad de México: El Colegio de México.

Yapu, M. (2010). La calidad y la evaluación a la luz de la nueva política educativa boliviana. Revista Iberoamericana de Educación, 53, 43-64.

Young, K. (2010). Progreso, patria y héroes. Una crítica del currículo de historia en México. Revista Mexicana de Investigación Educativa, 45, 599-620.

Young, M. (2008). Bringing knowledge back in: From social constructivism to social realism in the sociology of education. Londres: Routledge. 